\title{
La comparación de las desigualdades sociales entre Europa y América Latina: apreciaciones teóricas y metodológicas sobre un fenómeno complejo
}

\section{The comparison of social inequalities between Europe and Latin America: theoretical and methodological assessments of a complex} phenomenon

\section{Leticia Muñiz Terra}

lmunizterra@conicet.gov.ar

Centro Interdisciplinario de Metodología de las Ciencias Sociales. Instituto de Investigaciones en Humanidades y Ciencias Sociales (IdIHCS CONICET). Universidad Nacional de La Plata, Argentina

\section{Pablo Molina Derteano}

pablomd2009@gmail.com

Instituto de Investigaciones Gino Germani. CONICET - Universidad de Buenos Aires, Argentina

Recepción: 05 Mayo 2020

Aprobación: 30 Noviembre 2020

Publicación: 01 Febrero 2021

\begin{abstract}
Resumen: En este artículo se presenta un breve análisis de las contribuciones teóricas y empíricas que forman parte del presente dossier, enmarcándolas en las discusiones teóricas acerca de las desigualdades sociales y las comparaciones entre Europa y América Latina.
\end{abstract}

Palabras clave: Desigualdades Sociales, Métodos comparados, Europa, América Latina.

\begin{abstract}
In this article we present a brief analysis of the theoretical and empirical contributions included inthis dossier, framing them within a theory discusión about the social inequialities and the comparisons between Europe and América Latina.
\end{abstract}

Keywords: Social inequalities, Comparative methods, Europe, América Latina.
Cita sugerida: Muñiz Terra, L. y Molina Derteano, P. (2021). La comparación de las desigualdades sociales entre Europa y América Latina: apreciaciones teóricas y metodológicas sobre un fenómeno complejo. Cuestiones de Sociología, 24, e109. https://doi.org/10.24215/23468904e109
Las desigualdades sociales y sus múltiples manifestaciones son un problema estructural de los países latinoamericanos y europeos que las Ciencias Sociales se han encargado de estudiar en profundidad. Este fenómeno refleja, entre otras cuestiones: la falta de acceso a un empleo estable y con crecimiento personal 
y económico; las agudas desigualdades en el acceso a bienes y servicios -que se asocian a la destrucción de la llamada Sociedad Salarial-; los efectos de la precarización del empleo; la presencia de índices de desocupación de dos dígitos -que se agravan en el caso de las mujeres y los jóvenes-; la agudización de la segmentación del mercado laboral y su vinculación con circuitos socioeducativos excluyentes; los diferentes accesos a la tierra y la vivienda, la regresividad en la distribución del ingreso; la persistencia de las desigualdades entre las clases sociales; el acceso a consumos diferenciales, etc.

El estudio de estas distintas aristas de las desigualdades sociales se ha desarrollado en el campo sociológico a partir de diversas aproximaciones que enfocan su mirada tanto en las experiencias de los individuos -enfatizando la distribución de capacidades y recursos-, como en las pautas de relaciones e intercambios interinstitucionales desiguales, en las condiciones estructurales y asimétricas que se imponen a los actores sociales o en una articulación de estas miradas.

Las teorías centradas en las capacidades u oportunidades individuales enfocan su mirada en la distribución de diferentes atributos entre las personas y analizan cómo esta distribución incide sobre los resultados desiguales que se alcanzan en un contexto social dado. Para esta perspectiva, la desigualdad social es consecuencia de que la sociedad es un agregado de voluntades, acciones y decisiones individuales (Rawls, 1997), por lo cual, para alcanzar una mayor justicia e igualdad social, considera fundamental el fomento de las capacidades individuales (Sen, 1999).

Las miradas relacionales, en cambio, se han preocupado especialmente por el estudio de las relaciones o vínculos sociales que generan desigualdades. Estas pueden referir a las interacciones entre distintos actores que imponen fuertes restricciones (Goffman, 1981), o a pares categoriales distintivamente circunscriptos como mujer/varón, ciudadano/extranjero, aristócrata/plebeyo, etc., que pueden perdurar a lo largo de una carrera, una vida y una historia organizacional (Tilly, 2000).

Los estudios centrados en los condicionamientos estructurales han enfocado su mirada sobre las estructuras de distribución desigual de los beneficios y las cargas entre los diferentes sectores e individuos que conforman la sociedad. Sostienen, así, que la estructura económica y social propicia la ubicación de los individuos en distintos lugares de la sociedad y que el contexto macro-social explica fundamentalmente la existencia de la desigualdad social (CEPAL, 2010).

Las miradas integradoras han propuesto, por su parte, una perspectiva multidimensional y multiescalar, promoviendo una integración de las cuestiones macro, meso y microsociales (Therbon 2006; Reygadas, 2004; Wilkinson y Pickett, 2009; Dubet, 2011; Saraví, 2009; Savage, 2014) que en ocasiones puede incluir la mirada diacrónica; es decir, la comprensión de los fenómenos a lo largo del tiempo (Bertaux y Thompson, 1997; Muñiz Terra y Roberti, 2018).

Asimismo, la desigualdad puede analizarse de manera comparada entre países, regiones, grupos sociales o individuos. De hecho, la comparación ha sido una preocupación histórica de la sociología que ha ido ganando importancia e interés. Emile Durkheim, uno de los padres fundadores de las ciencias sociales y referente teórico de la sociología, defendiendo la importancia de la comparación sostuvo en sus escritos que "la sociología comparativa no es una rama particular de la 
sociología, desde el momento en que deja de ser sólo descriptiva y aspira a explicar los hechos" (1996, p. 97). Estas ideas fueron luego recuperadas por múltiples disciplinas de las ciencias sociales más allá de la sociología, como la historia, la ciencia política y la antropología (Edwards, 1927; Sckopol y Somers, 1980; Sartori, 1984; Pérez, 2007, etc.).

Estas aproximaciones han definido, en líneas generales, la perspectiva comparativa como una aproximación que se preocupa por la descripción y la explicación de problemas de la realidad social, analizando las condiciones y los resultados semejantes y diferentes (usualmente estos últimos) entre unidades sociales grandes como naciones, sociedades y culturas, y/ o unidades más pequeñas como individuos, grupos sociales e instituciones (Przeworsky y Teune, 1970; Smelser, 2003).

En particular, algunos autores (Przeworsky y Teune, 1970; Liphjar, 1971; Collier, 1994; Cais, 1997; Colino, 2007; Ariza y Gandini, 2012) han señalado que la realidad social es infinitamente diversa y que debe ser estudiada a partir de investigaciones comparativas. Para ellos, la comparación puede abarcar diferentes cuestiones, pues puede incluir la comparación de problemáticas generales de dos o más entidades macrosociales o microsociales, o de dos o más momentos en la historia de una sociedad, y tiene como objetivo primordial analizar sus semejanzas y diferencias e indagar sus causas.

La preocupación por las desigualdades sociales y la comparación animan el conjunto de artículos que este dossier compila y que han sido escritos en el marco del proyecto europeo International Network for Comparative Analysis of Social Inequalities (INCASI) dirigido por el Dr. Pedro López Roldán. El proyecto de la Red INCASI estuvo integrado por veinte universidades de cinco países europeos (España, Italia, Francia, Reino Unido y Finlandia) y de cinco países latinoamericanos (Argentina, Uruguay, Chile, Brasil y México). Recibió apoyo del programa europeo Horizon 2020, dentro de las Marie SkłodowskaCurie Actions, Research and Innovation Staff Exchange (Grant Agreement GA-691004), y dio lugar a investigaciones comparadas entre los años 2016 y 2019.

Los textos que se presentan en este dossier son, así, el resultado de los intercambios y experiencias de investigación y formación conjunta desarrollados en el marco de INCASI y buscan contribuir a comprender múltiples problemáticas de las desigualdades sociales a partir de análisis comparativos entre países de distintos continentes, países de un mismo continente y/o grupos sociales distintos al interior de los países, desplegando modelos analíticos que incluyen tanto metodologías cuantitativas como cualitativas y mixtas.

En la sección "Artículos de investigación" los estudios cuantitativos se plantean las tensiones referentes a la disponibilidad de bases de datos, sus criterios de construcción y las potencialidades y limitaciones en la comparabilidad. Los artículos de Zawadsky; Rodríguez Espínola, Filgueira, Sartí; y Molina Derteano proveen, así, ejemplos de esos debates en comparaciones entre países europeos y latinoamericanos en los dos primeros o entre países latinoamericanos en el tercero. Aportan a fortalecer la construcción de enunciados teóricos y empíricos que surgen de las evidencias estadísticas y que son punto de partida para futuros estudios. Las investigaciones cualitativas ponen de relieve, por su parte, la potencialidad de la recuperación de la mirada de los actores sociales para 
comprender en profundidad las desigualdades sociales en términos comparados. Los artículos de Domínguez Amorós y Aravena; Muñiz Terra; y Ferrari dan cuenta, así, de la relevancia de realizar comparaciones cualitativas entre clases sociales y de enfocar la mirada en grupos específicos y estudiar comparativamente lo que acontece en su interior.

La sección "Notas de investigación" presenta un texto que desarrolla una perspectiva innovadora en el diseño de una investigación comparativa entre países de Europa y América Latina, con la movilidad social y las trayectorias laborales como ejes analíticos. Segura Carrillo propone en sus notas la implementación de una mirada mixta para el estudio de las desigualdades sociales comparadas que, de acuerdo con su mirada, permite alcanzar una mayor comprensión y profundización de los fenómenos sociales.

En la sección "Debate", se recuperan concretamente algunas discusiones en torno a distintas expresiones de las desigualdades comparadas, como lo post/ decolonial, la interseccionalidad, el feminismo, las migraciones y el acceso, uso y apropiación de las tecnologías digitales. Souto García, Ambort y Lemus presentan así los debates temáticos existentes y su relación con las problemáticas de las desigualdades sociales comparadas reponiendo la relevancia de profundizar las miradas cualitativas comparativas.

Para la sección “Entrevista” fue convocado el Dr. Pedro López Roldán, director del proyecto INCASI, quién en la conversación que mantuvo con Chávez Molina y Roberti detalló su trayectoria académica y sus principales líneas de investigación, comentó los enfoques teóricos y metodológicos que desde su parecer son los más apropiados para analizar la persistencia de las desigualdades sociales en la actualidad, y desarrolló los desafíos que implica desarrollar una perspectiva comparada sobre las desigualdades entre América Latina y Europa.

En la sección "Lecturas", Atairo y Piovani reseñan el libro Towards a Comparative Analysis of Social Inequalities between Europe and Latin America publicado por la editorial Springer, que es el resultado de las discusiones e intercambios de los investigadores responsables de las veinte universidades de la Red INCASI y que fue coordinado por López Roldán y Fachelli. Asimismo, en esta sección Cáneva reseña el libro La ¿nueva? estructura social de América Latina. Cambios y persistencias después de la ola de gobiernos progresistas, de Benza y Kessler, publicado por la editorial Siglo XXI.

Para concluir, quisiéramos agradecer a varias personas e instituciones que hicieron posible este dossier. En primer lugar vaya nuestro reconocimiento a la Dra. Sandra Fachelli y al Dr. Pedro López Roldán, y por su intermedio a la Red INCASI, por su permanente estímulo para que este monográfico diera a luz; en segundo lugar quisiéramos agradecer a los investigadores de la RED INCASI que se sumaron a la aventura de reflexionar y poner en sus textos apreciaciones e investigaciones sobre las desigualdades sociales comparadas; en tercer lugar agradecemos a los evaluadores anónimos de universidades de ambos lados del Atlántico, por su compromiso y dedicación para realizar los referatos y sugerencias que permitieron mejorar y dar solidez a los textos que aquí se presentan; y finalmente, quisiéramos agradecer al Centro Interdisciplinario de Metodología de las Ciencias Sociales (CIMeCS-IdIHCS. UNLP-CONICET) y al Departamento de Sociología de la Facultad de Humanidades y Ciencias de la Educación de la Universidad Nacional de La Plata, instituciones que brindaron 
un gran apoyo para la elaboración de este dossier que con entusiasmo invitamos a leer y compartir.

\section{Referencias}

Ariza, M. y Gandini, L. (2012). El análisis comparativo cualitativo como estrategia metodológica. En M. Ariza., Métodos cualitativos y su aplicación empírica: por los caminos de la investigación sobre migración internacional (pp. 497-537). México D. F.: UNAM.

Bertaux, D. y Thompson, P. (1997). Introduction. En Autor, Pathways to social class (pp 1-31). Nueva York: Oxford University Press.

Cais, J. (1997). Metodología del análisis comparativo. Madrid: Centro de Investigaciones Sociológicas - CIS. Cuadernos Metodológicos, 21.

CEPAL (2010). Heterogeneidad estructural y brechas de productividad: de la fragmentación a la convergencia. En CEPAL. Documento La hora de la igualdad. Brechas para cerrar, caminos por abrir. (pp. 1-289) Santiago de Chile: Editorial CEPAL.

Colino, C. (2007). Método comparativo. En Autor, Diccionario Crítico de Ciencias Sociales (pp. 13-32). México D. F.: Plaza Valdés.

Collier, D. (1994). El método comparativo: dos décadas de cambios. En G. Sartori, La comparación en las ciencias sociales (pp. 21-46), Madrid: Alianza.

Dubet, F. (2011). La experiencia sociológica. Buenos Aires: Gedisa.

Durkheim, E. (1996). Las nuevas reglas del método sociológico. México D. F.: Ed. Coyoacán.

Edwards, L. P. (1927). The natural history of revolution. Chicago: University of Chicago Press.

Goffman, E. (1981). La presentación de la persona en la vida cotidiana. Buenos Aires: Amorrortu.

Muñiz Terra, L. y Roberti, E. (2018). "Las tramas de la desigualdad social desde una perspectiva comparada: hacia una reconstrucción de las trayectorias laborales de jóvenes de clases medias y trabajadoras". Revista Estudios del trabajo, $\mathrm{N}^{\circ} 55$, pp. $1-32$.

Pérez, A. (2007). El método comparativo: fundamentos y desarrollos recientes. Recuperado de http://www.pitt.edu/ asp27/USAL/2007.Fundamentos.pdf

Przeworsky, A. y Teune, H. (1970). The Logic of Comparative Social Inquiry. Nueva York: Wiley ed.

Rawls, J. (1997). Teoría de la justicia. México D. F.: Fondo de Cultura Económica.

Reygadas, L. (2004). Las redes de la desigualdad: un enfoque multidimensional. Politica y cultura, (num 22), 7-25. Recuperado de http://www.scielo.org.mx/scielo.php?s cript $=$ sci_arttext\&pid=S0188-77422004000200002\&lng=es\&tlng=es.

Saraví, G. (2009).Transiciones vulnerables: Juventud, desigualdad y exclusión en México . México D. F.: CIESAS.

Sartori, G. (1984). Lapolítica, lógica y método en las ciencias sociales. México D. F.: Fondo de Cultura Económica.

Savage, M. (2014). A new modelo of social class? Findings from BBC's great British class survey experiment. Sociology, 47(2).

Sen, A. (1999). Nuevo examen de la desigualdad. Madrid: Alianza Editorial. 
Skocpol, T. y Somers, M (1980). The uses of comparative history in macrosocial inquiry. Comparative Studies in Society and History, 22(2).

Smelser, N. (2003). On comparative analysis, interdisciplinarity and internationalization in Sociology. Studies in Comparative International Development, 1(40), 39-42.

Therborn, G. (2006). Inequalities and Latin America from the Enlightenment to the 21st Century. Documento de trabajo $N^{\circ} 1$. Desigualdades.net. Recuperado de http://www.desigualdades.net/Working_Papers/Search-Working-Papers/W orking-Paper-1__Inequalities-and-Latin-America_/index.html

Tilly, C. (2000). La desigualdad persistente. Buenos Aires: Manantial.

Wilkinson, R. y Pickett, K. (2009): The Spirit Level. Londres: Allen Lane. 